\title{
A systematic review of randomized controlled trials exploring the effect of immunomodulative interventions on infection, organ failure, and mortality in trauma patients
}

Nicole E Spruijt, Tjaakje Visser, Luke PH Leenen*

\begin{abstract}
Introduction: Following trauma, patients may suffer an overwhelming pro-inflammatory response and immune paralysis resulting in infection and multiple organ failure (MOF). Various potentially immunomodulative interventions have been tested. The objective of this study is to systematically review the randomized controlled trials (RCTs) that investigate the effect of potentially immunomodulative interventions in comparison to a placebo or standard therapy on infection, MOF, and mortality in trauma patients.

Methods: A computerized search of MEDLINE, the Cochrane CENTRAL Register of Controlled Trials, and EMBASE yielded 502 studies, of which 18 unique RCTs were deemed relevant for this study. The methodological quality of these RCTs was assessed using a critical appraisal checklist for therapy articles from the Centre for Evidence Based Medicine. The effects of the test interventions on infection, MOF, and mortality rates and inflammatory parameters relative to the controls were recorded.
\end{abstract}

Results: In most studies, the inflammatory parameters differed significantly between the test and control groups. However, significant changes in infection, MOF, and mortality rates were only measured in studies testing immunoglobulin, IFN- $\gamma$, and glucan.

Conclusions: Based on level $1 \mathrm{~b}$ and $2 \mathrm{~b}$ studies, administration of immunoglobulin, IFN- $\gamma$, or glucan have shown the most promising results to improve the outcome of trauma patients.

\section{Introduction}

Trauma remains the leading cause of death in people under the age of 40 years [1], with multiple organ failure (MOF) accounting for $27.5 \%$ of deaths among trauma patients [2]. MOF can be a result of an early over-reaction of the immune system or a late immune paralysis [3]. Several groups have reviewed the changes that occur in the immune system as a result of injury and concluded that pro- and anti-inflammatory reactions play a role in the development of MOF [4-7]. Early MOF, which develops within the first three days after injury without signs of infection, is attributed to an overwhelming leukocyte driven pro-inflammatory

\footnotetext{
* Correspondence: L.P.H.Leenen@umcutrecht.nl

Department of Surgery, University Medical Centre Utrecht, H.P. G04.228, Heidelberglaan 100, 3584 GX Utrecht, The Netherlands

(1)
}

response clinically defined as a systemic inflammatory response syndrome (SIRS). Late MOF, on the other hand, is most often associated with infection and occurs more than three days after injury. Late MOF seems to be the result an inadequate specific immune response with diminished antigen presentation, referred to as compensatory anti-inflammatory response syndrome (CARS). Many argue that SIRS and CARS occur simultaneously as a mixed antagonistic response syndrome (MARS) $[4,6]$ and therefore both reactions contribute to the occurrence of infection, sepsis, and MOF.

This knowledge needs to be applied. Which interventions attenuate both the hyper-inflammatory response and immune paralysis and subsequently improve the clinical outcome in trauma patients? Montejo et al. [8] have systematically reviewed the effect of immunonutrition on
C Biomed Central 
clinical outcome in trauma patients. Although immunonutrition shortened the time of mechanical ventilation and ICU stay, and resulted in a lower incidence of bacteremias and intra-abdominal infections, the incidence of nosocomial pneumonia, wound infection, urinary tract infection, sepsis, and mortality remain unchanged. Other interventions are needed.

The objective of this paper is to systematically review the randomized controlled trials (RCTs) that investigate the effect of non-nutritional potential immunomodulative interventions in comparison to a placebo or standard therapy on infection, MOF, and mortality in trauma patients.

\section{Materials and methods}

\section{Search}

Studies were found via computerized searches of the MEDLINE and EMBASE databases and the Cochrane CENTRAL Register of Controlled Trials. The search syntax included synonyms of trauma (trauma", injur*), immunomodulation (immun*, inflammat*), and clinical outcome (infectio*, "organ failure", mortality, surviv*) in the titles, abstracts, and keywords areas. Limits were set to retrieve only studies on humans with high-quality design (meta-analyses, systematic reviews, Cochrane reviews, RCTs, and clinical trials). No limits were imposed on either publication date or language.

\section{Selection}

The search hits were screened for relevance by two authors. Studies were deemed relevant when they investigated the effect of a potentially immunomodulative intervention on clinical outcome in trauma patients. Therefore, studies including patients other than trauma patients (for example, other ICU patients), patients with specific isolated injury (for example, isolated injury to the head or an extremity), or patients with thermal injuries were excluded. Furthermore, patients needed to be randomly allocated to receive a potentially immunomodulative intervention, standard therapy, or a placebo. As the effect of immunonutrition has already been systematically reviewed, studies implementing immunonutrition were excluded. To assess the efficacy of the interventions, only studies reporting clinical outcomes were included. References of the relevant studies were checked for other relevant articles that might have been missed in the computerized search.

\section{Quality assessment}

The methodological quality of each of the studies for which the full text was available was assessed using a checklist for therapy articles from the Centre for Evidence Based Medicine $[9,10]$. One point was accredited for each positive criterion: the study participants were randomized; the study groups had similar characteristics at baseline; the groups were treated equally except for the test intervention; all patients were accounted for; outcome assessors were blinded to the intervention or used well-defined outcome criteria; and outcomes were compared on an intention-to-treat basis.

\section{Data abstraction}

Data abstraction was completed independently. The studies were searched for patient characteristics (number, age, and injury severity score (ISS)), details of the intervention (test, control, delivery route, and duration) and length of follow-up during which outcome variables were measured. Outcome variables included in the analysis were: infections, overall or specified; MOF or mortality; and inflammatory parameters, cellular or humoral. Definitions of infections given by authors were used, including major and minor infections, pneumonia, sepsis, meningitis, surgical site infections, urinary tract infections, and intra-abdominal abscesses. MOF was defined by MOF scores given by the authors. The efficacy of interventions intended to attenuate the hyperinflammatory response were compared with those intended to reduce the immune paralysis. Interventions that altered the release of pro-inflammatory cytokines (IL-1 $\beta$, IL-6, IL- 8 , TNF- $\alpha$ ), active complement factors, leukocyte count, or leukocyte-derived cytotoxic mediators were considered modulators of SIRS. Interventions that altered the release of anti-inflammatory cytokines (IL-10, IL-1RA), antigen-presenting capacity, or bactericidal capacity were considered modulators of CARS.

\section{Results}

\section{Search and selection}

After filtering out duplicate studies retrieved from the databases, 502 potentially relevant studies were assessed. Studies were excluded that did not include only trauma patients (444), tested interventions that were not intended to immunomodulate (10), studied the effect of immunonutrition (20), did not report clinical outcome (4), or were non-systematic reviews (5) (Figure 1). The full text was not available for two studies $[11,12]$. By checking references of the relevant studies, three other relevant studies were found that were missed in the computerized search because the keywords were not included in the titles or abstracts [13-15]. Two articles by Seekamp et al. $[16,17]$ and two articles by Dries et al. $[13,18]$ report on the same study. Therefore, 18 unique RCTs that met the inclusion and exclusion criteria were available for analysis.

\section{Quality assessment}

Using the checklist for therapy articles from the Centre for Evidence Based Medicine [9], all RCTs scored four 


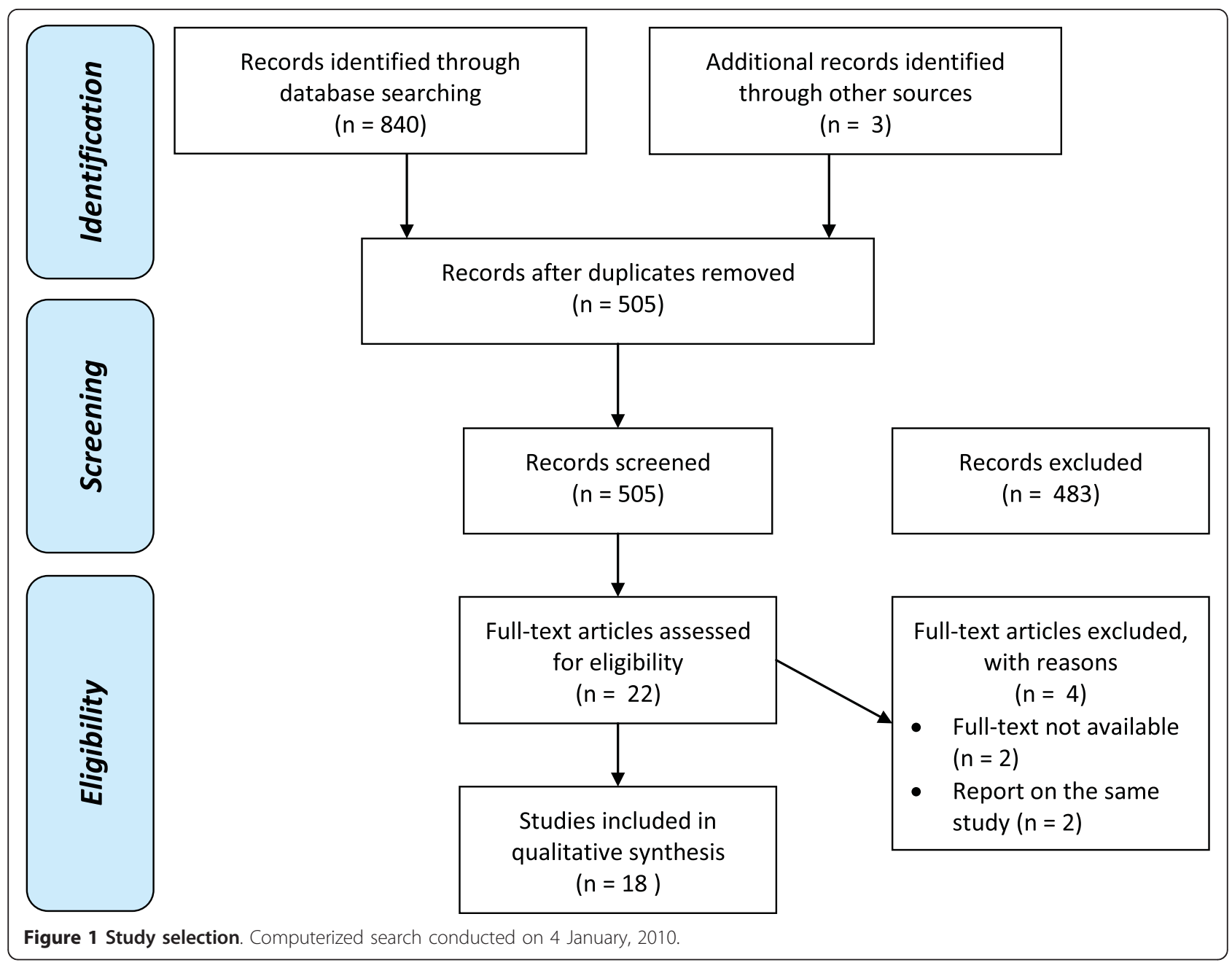

to six out of a maximum six points (Table 1). Points were lost because the study groups were dissimilar at baseline and/or patients dropped out that were not analyzed on an intention-to-treat basis. Studies scoring a full six points were deemed high-quality RCTs reporting $1 \mathrm{~b}$ level of evidence [10]. Studies scoring four or five points were deemed of lesser quality and thus reporting $2 \mathrm{~b}$ level of evidence. Data from all studies were used to determine the effect of potential immunomodulative interventions on clinical outcome in trauma patients.

\section{Study characteristics}

A comparison of the study characteristics of the 18 RCTs reveals marked inter-trial heterogeneity of patients and interventions (Table 2). The number of patients included in the trials ranged from 16 to 268, with five trials studying over 100 patients [19-23]. Of the smaller trials, six were pilot studies [14,24-27]. Three of the trials were phase II trials primarily powered to test dosage and safety, not efficacy $[16,23,24]$. Patient ages ranged between 13 and 90 years, with the mean age in the $30 \mathrm{~s}$ or low $40 \mathrm{~s}$ for all studies except those of Rizoli et al. [27] and Seekamp et al. $[16,17]$ in which the mean age was nearer 50 years. Similarly, the ISS ranged from 0 to 75 , with the mean ISS in the $20 \mathrm{~s}$ or low $30 \mathrm{~s}$ for most studies. The studies by Nakos et al. [26] and Waydhas et al. [28] averaged more severely injured patients.

Interventions were intended to attenuate the early overwhelming inflammatory response and diminish the immune paralysis. As many trauma patients are plagued by infections, researchers aimed to augment the host's inflammatory response by stimulating macrophages with glucan [29,30], activating monocytes with dextran [14], upregulating human leukocyte antigen (HLA)-DR expression with interferon (IFN) $-\gamma[18,22,26,31]$, and providing immunoglobulins $[20,32]$. As hyper-inflammation causes injury, researchers aimed to taper the host's inflammatory response by infusing leuko-reduced blood [21], prostaglandin E1 [15], antioxidants [25], and antithrombin III [28], which, by blocking thrombin, decreases IL-8 production and sequestration of 
Table 1 Quality assessment

\begin{tabular}{|c|c|c|c|c|c|c|c|c|}
\hline Study & $\begin{array}{c}\text { Patients } \\
\text { randomized }\end{array}$ & $\begin{array}{l}\text { Groups similar } \\
\text { at baseline }\end{array}$ & $\begin{array}{l}\text { Groups } \\
\text { treated } \\
\text { equally }\end{array}$ & $\begin{array}{l}\text { All patients } \\
\text { accounted for }\end{array}$ & $\begin{array}{l}\text { Assessor blinded } \\
\text { or objective }\end{array}$ & $\begin{array}{l}\text { Intention to } \\
\text { treat analysis }\end{array}$ & $\begin{array}{l}\text { TOTAL } \\
(\max 6)\end{array}$ & $\begin{array}{l}\text { Level of } \\
\text { Evidence }\end{array}$ \\
\hline $\begin{array}{l}\text { Browder et al, } \\
1990 \text { [29] }\end{array}$ & 1 & 1 & 1 & 1 & 1 & 1 & 6 & $1 b$ \\
\hline $\begin{array}{l}\text { Bulger et al, } \\
2008[19]\end{array}$ & 1 & 1 & 1 & 1 & 1 & 1 & 6 & $1 b$ \\
\hline $\begin{array}{l}\text { Croce et al, } \\
1998 \text { [24] }\end{array}$ & 1 & $0^{\circ}$ & 1 & 1 & 1 & 1 & 5 & $2 b$ \\
\hline $\begin{array}{l}\text { de Felippe } \\
\text { et al, } 1993 \text { [30] }\end{array}$ & 1 & 1 & 1 & 1 & 1 & 0 & 5 & $2 b$ \\
\hline $\begin{array}{l}\text { Douzinas et al, } \\
2000 \text { [32] }\end{array}$ & 1 & $0^{*}$ & 1 & 1 & 1 & 0 & 4 & $2 b$ \\
\hline $\begin{array}{l}\text { Dries et al, } \\
1998[18]\end{array}$ & 1 & 1 & 1 & 1 & 1 & 0 & 5 & $2 b$ \\
\hline $\begin{array}{l}\text { Glinz et al, } \\
1985[20]\end{array}$ & 1 & 1 & 1 & 1 & 1 & 1 & 6 & $1 b$ \\
\hline $\begin{array}{l}\text { Livingston et al, } \\
1994 \text { [31] }\end{array}$ & 1 & 1 & 1 & 1 & 1 & 1 & 6 & $1 b$ \\
\hline $\begin{array}{l}\text { Marzi et al, } \\
1993 \text { [25] }\end{array}$ & 1 & 1 & 1 & 1 & 1 & 1 & 6 & $1 b$ \\
\hline $\begin{array}{l}\text { Miller \& Lim, } \\
1985 \text { [14] }\end{array}$ & 1 & n.r. & 1 & 1 & 1 & 0 & 4 & $2 b$ \\
\hline $\begin{array}{l}\text { Nakos et al, } \\
2002[26]\end{array}$ & 1 & 1 & 1 & 1 & 1 & 1 & 6 & $1 b$ \\
\hline $\begin{array}{l}\text { Nathens et al, } \\
2006 \text { [21] }\end{array}$ & 1 & 1 & 1 & 1 & 1 & 1 & 6 & $1 b$ \\
\hline $\begin{array}{l}\text { Polk et al, } \\
1992 \text { [22] }\end{array}$ & 1 & $0^{\circ}$ & 1 & 1 & 1 & 1 & 5 & $2 b$ \\
\hline $\begin{array}{l}\text { Rhee et al, } \\
2000 \text { [23] }\end{array}$ & 1 & 0 & 1 & 1 & 1 & 1 & 5 & $2 b$ \\
\hline $\begin{array}{l}\text { Rizoli et al, } \\
2006 \text { [27] }\end{array}$ & 1 & 0 & 1 & 1 & 1 & 0 & 4 & $2 b$ \\
\hline $\begin{array}{l}\text { Seekamp et al, } \\
2004 \text { [16] }\end{array}$ & 1 & 1 & 1 & 1 & 1 & 1 & 6 & $1 b$ \\
\hline $\begin{array}{l}\text { Vassar et al, } \\
1991[15]\end{array}$ & 1 & 1 & 1 & 1 & 1 & 1 & 6 & $1 b$ \\
\hline $\begin{array}{l}\text { Waydhas et al, } \\
1998 \text { [28] }\end{array}$ & 1 & 1 & 1 & 1 & 1 & 0 & 5 & $2 b$ \\
\hline
\end{tabular}

$1=$ yes; $0=$ no; n.r. $=$ not reported, the test group was older; ${ }^{*}=$ the test group had a higher injury severity score, which was corrected for using a multiple regression model.

neutrophils. By blocking a neutrophil receptor that binds to endothelium (CD18) [23] or an adhesion molecule (L-selectin) [16] with an antibody, researchers hoped to prevent neutrophils from extravasating and causing reperfusion injury after hemorrhagic shock. Perflubron is attributed with anti-inflammatory properties because macrophages exposed to it demonstrate significantly less hydrogen peroxide superoxide anion and production [24]. Most of the control groups were given a placebo [15-18,20,22,23,25-32] and four received only standard treatment $[14,19,21,24]$. The interventions were administered intravenously [14-17,19-21,23,25,27-30,32], subcutaneously $[18,22,31]$, or via inhalation $[24,26]$. Interventions were initiated as soon as possible after injury by ambulance personnel [19] or as late as
145 hours after hospital admission [30]. The duration of the intervention differed from a single dose to 28 days. The length of follow-up ranged from 10 to 90 days.

\section{Outcomes}

Among the outcome variables, most of the significant differences between the test and control groups were in inflammatory parameters, suggesting attenuation of SIRS, CARS, or both (Table 3). Only monoclonal antibodies against CD18 [23] exacerbated SIRS and hypertonic saline with dextran had a mixed effect on CARS [27]. Significant changes in infection and mortality rates were only measured in the studies testing IFN- $\gamma[18,26]$, immunoglobulin $[20,32]$, and glucan $[29,30]$. These were not the most recently published or largest studies, nor the studies with 
Table 2 Study characteristics

\begin{tabular}{|c|c|c|c|c|c|c|c|c|c|}
\hline \multirow[t]{2}{*}{ Study } & \multicolumn{3}{|c|}{ Patients } & \multicolumn{5}{|c|}{ Intervention } & \multirow[b]{2}{*}{$\begin{array}{l}\text { Length } \\
\text { of } \\
\text { follow- } \\
\text { up }\end{array}$} \\
\hline & $\mathbf{n}$ & $\begin{array}{l}\text { Age } \\
\text { (range) }\end{array}$ & $\begin{array}{l}\text { ISS (range, } \\
\pm \mathrm{SD} \text { ) }\end{array}$ & Test & Control & Delivery & Initiation & Duration & \\
\hline $\begin{array}{l}\text { Browder } \\
\text { et al, } 1990 \\
{[29]}\end{array}$ & 38 & $34(18-65)$ & $24(8-41)$ & Glucan & placebo (saline) & i.v. & $\begin{array}{l}\text { after exploratory } \\
\text { laparotomy or } \\
\text { thoracotomy }\end{array}$ & 7 days & 10 days \\
\hline $\begin{array}{l}\text { Bulger } \\
\text { et al, } 2008 \\
\text { [19] }\end{array}$ & 209 & $38(13-90)$ & $28(0-75)$ & $\begin{array}{l}\text { Hypertonic saline + } \\
\text { Dextran }\end{array}$ & $\begin{array}{l}\text { Lactated Ringer } \\
\text { solution }\end{array}$ & i.v. & $\begin{array}{l}\text { initial reperfusion } \\
\text { fluid }\end{array}$ & single dose & 28 days \\
\hline $\begin{array}{l}\text { Croce et al, } \\
1998 \text { [24] }\end{array}$ & 16 & $32(15-75)$ & 29 & $\begin{array}{l}\text { Partial liquid } \\
\text { ventilation with } \\
\text { perflubron }\end{array}$ & $\begin{array}{l}\text { Conventional } \\
\text { mechanical } \\
\text { ventilation }\end{array}$ & Inhaled & day of admission & 4 days & $\begin{array}{l}\text { hospital } \\
\text { discharge }\end{array}$ \\
\hline $\begin{array}{l}\text { de Felippe } \\
\text { et al, } 1993 \\
\text { [30] }\end{array}$ & 41 & $35(16-76)$ & $n . r^{*}$ & Glucan & placebo & i.v. & $\begin{array}{l}12-145 \mathrm{hr} \text { (mean } \\
46.2 \mathrm{hr} \text { ) after } \\
\text { admission }\end{array}$ & 3-17 days & $\begin{array}{l}\text { hospital } \\
\text { discharge }\end{array}$ \\
\hline $\begin{array}{l}\text { Douzinas } \\
\text { et al, } 2000 \\
\text { [32] }\end{array}$ & 39 & 32 & $24(16-50)$ & Immunoglobulin & placebo (albumin) & i.v. & $\begin{array}{l}12 \mathrm{hr} \text { after } \\
\text { admission }\end{array}$ & 6 days & $\begin{array}{l}\text { hospital } \\
\text { discharge }\end{array}$ \\
\hline $\begin{array}{l}\text { Dries et al, } \\
1998 \text { [18] }\end{array}$ & 73 & 31 & $34(21-59)$ & rhIFN- $\gamma$ & placebo & s.c. & $\begin{array}{l}\text { within } 30 \mathrm{hr} \text { of } \\
\text { injury }\end{array}$ & $\begin{array}{l}21 \text { days or } \\
\text { hospital } \\
\text { discharge }\end{array}$ & 60 days \\
\hline $\begin{array}{l}\text { Glinz et al, } \\
1985 \text { [20] }\end{array}$ & 150 & $39(15-78)$ & $30(9-66)$ & Immunoglobulin & placebo (albumin) & i.v. & $\begin{array}{l}\text { within } 24 \mathrm{hr} \text { of } \\
\text { starting mechanical } \\
\text { ventilation }\end{array}$ & 12 days & 42 days \\
\hline $\begin{array}{l}\text { Livingston } \\
\text { et al, } 1994 \\
\text { [31] }\end{array}$ & 98 & $30(>16)$ & $30( \pm 8)$ & rhIFN- $\gamma$ & placebo & S.c. & day of admission & 10 days & 30 days \\
\hline $\begin{array}{l}\text { Marzi et al, } \\
1993 \text { [25] }\end{array}$ & 24 & $32(18-57)$ & $34(27-57)$ & $\begin{array}{l}\text { superoxide } \\
\text { dismutase }\end{array}$ & placebo (sucrose) & i.v. & $\begin{array}{l}\text { within } 48 \mathrm{hr} \text { of } \\
\text { injury }\end{array}$ & 5 days & 14 days \\
\hline $\begin{array}{l}\text { Miller \& } \\
\text { Lim, } 1985 \\
{[14]}\end{array}$ & 28 & n.r. & $>10$ & $\begin{array}{l}\text { Dextran + standard } \\
\text { treatment }\end{array}$ & standard treatment & i.v. & $\begin{array}{l}\text { within } 12 \mathrm{hr} \text { of } \\
\text { admission }\end{array}$ & 5 days & 4 weeks \\
\hline $\begin{array}{l}\text { Nakos } \\
\text { et al, } 2002 \\
\text { [26] }\end{array}$ & 21 & $49(35-67)$ & $41(24-62)$ & rhIFN- $\gamma$ & placebo & inhaled & $\begin{array}{l}\text { 2nd or 3rd day } \\
\text { after admission }\end{array}$ & 7 days & $\begin{array}{l}\text { hospital } \\
\text { discharge }\end{array}$ \\
\hline $\begin{array}{l}\text { Nathens } \\
\text { et al, } 2006 \\
{[21]}\end{array}$ & 268 & $42(>17)$ & $24( \pm 11)$ & $\begin{array}{l}\text { Leukoreduced }(<5 \times \\
10 \wedge 6 \mathrm{WBC}) \mathrm{RBC} \\
\text { transfusion }\end{array}$ & $\begin{array}{l}\text { Nonleukoreduced ( } 5 \\
\times 10 \wedge 9 W B C) \text { RBC } \\
\text { transfusion }\end{array}$ & i.v. & $\begin{array}{l}\text { within } 24 \mathrm{hr} \text { of } \\
\text { injury }\end{array}$ & 28 days & 28 days \\
\hline $\begin{array}{l}\text { Polk et al, } \\
1992 \text { [22] }\end{array}$ & 193 & $32(>15)$ & $33(>20)$ & rhIFN- $\gamma$ & placebo & s.c. & day of admission & 10 days & 90 days \\
\hline $\begin{array}{l}\text { Rhee et al, } \\
2000 \text { [23] }\end{array}$ & 116 & $40(>18)$ & $20( \pm 11)$ & rhMAbCD18 & placebo & i.v. & day of admission & single dose & $\begin{array}{l}\text { hospital } \\
\text { discharge }\end{array}$ \\
\hline $\begin{array}{l}\text { Rizoli et al, } \\
2006 \text { [27] }\end{array}$ & 24 & $48(>16)$ & $26( \pm 11)$ & $\begin{array}{l}\text { Hypertonic saline + } \\
\text { Dextran }\end{array}$ & placebo (saline) & i.v. & $\begin{array}{l}\text { upon arrival in de } \\
\text { emergency } \\
\text { department }\end{array}$ & single dose & $\begin{array}{l}\text { hospital } \\
\text { discharge }\end{array}$ \\
\hline $\begin{array}{l}\text { Seekamp } \\
\text { et al, } 2004 \\
{[16]}\end{array}$ & 84 & $36(17-72)$ & $32(17-59)$ & $\begin{array}{l}\text { Anti-L-Selectin } \\
\text { (Aselizumab) }\end{array}$ & placebo & i.v. & within $6 \mathrm{hr}$ of injury & single dose & 42 days \\
\hline $\begin{array}{l}\text { Vassar } \\
\text { et al, } 1991 \\
{[15]}\end{array}$ & 48 & 36 & $31( \pm 3)$ & Prostaglandin E1 & placebo & i.v. & $\begin{array}{l}24-48 \mathrm{hr} \text { after } \\
\text { hospital admission }\end{array}$ & 7 days & $\begin{array}{l}\text { hospital } \\
\text { discharge }\end{array}$ \\
\hline $\begin{array}{l}\text { Waydhas } \\
\text { et al, } 1998 \\
{[28]}\end{array}$ & 40 & $33(18-70)$ & $41( \pm 13)$ & Antithrombin III & placebo (albumin) & i.v. & within $6 \mathrm{hr}$ of injury & 4 days & $\begin{array}{l}\text { hospital } \\
\text { discharge }\end{array}$ \\
\hline
\end{tabular}

IFN, interferon; ISS, injury severity score; i.v., intravenous; n, number; n.r., not reported; RBC, red blood cell; s.c., subcutaneous; WBC, white blood cell; * Trauma score 10 , denoted as 'severe multiple trauma'. 
Table 3 Study results

\begin{tabular}{|c|c|c|c|c|c|c|c|c|}
\hline \multirow{2}{*}{$\begin{array}{l}\text { Test } \\
\text { intervention }\end{array}$} & & \multirow[b]{2}{*}{ Study } & \multicolumn{2}{|l|}{ Infection } & \multicolumn{2}{|c|}{ MOF, Mortality } & \multicolumn{2}{|l|}{ Inflammation } \\
\hline & & & $\begin{array}{l}\text { Test group (relative to } \\
\text { control) }\end{array}$ & Effect & $\begin{array}{l}\text { Test group } \\
\text { (relative to } \\
\text { control) }\end{array}$ & Effect & $\begin{array}{l}\text { Test group (relative to } \\
\text { control) }\end{array}$ & Effect \\
\hline \multirow[t]{11}{*}{$\begin{array}{l}\text { Reduce } \\
\text { immune } \\
\text { paralysis }\end{array}$} & $\begin{array}{l}\text { Plasma } \\
\text { expander }\end{array}$ & $\begin{array}{l}\text { Miller \& } \\
\text { Lim, } 1985 \\
{[14]}\end{array}$ & & & $\begin{array}{l}\text { Mortality } 0 \text { vs } \\
0 \text { n.s. }\end{array}$ & $\begin{array}{l}\text { No } \\
\text { effect }\end{array}$ & immune reactive capacity n.s. & $\begin{array}{l}\text { No } \\
\text { effect }\end{array}$ \\
\hline & & $\begin{array}{l}\text { Rizoli et al, } \\
2006 \text { [27] }\end{array}$ & $\begin{array}{l}\text { pneumonia } \\
0.5 \% \text { vs } 0.5 \% \text { n.s. }\end{array}$ & $\begin{array}{l}\text { No } \\
\text { effect }\end{array}$ & $\begin{array}{l}\text { Mortality } 0 \text { vs } \\
14.3 \% \text { n.s., MOF } \\
\text { score } 1.68 \text { vs } 1.9 \\
\text { n.s. }\end{array}$ & $\begin{array}{l}\text { No } \\
\text { effect }\end{array}$ & $\begin{array}{l}\text { WBC n.s.; decreased toward } \\
\text { normal: CD11b, CD62L, CD16, } \\
\text { and TNF } \alpha \text {; increased toward } \\
\text { normal: CD14, IL-1RA, and IL-10 } \\
\text { all } P<0.05\end{array}$ & $\begin{array}{l}\text { SIRS } \downarrow \\
\text { and } \\
\text { CARS } \downarrow \uparrow\end{array}$ \\
\hline & & $\begin{array}{l}\text { Bulger } \\
\text { et al, } 2008 \\
{[19]}\end{array}$ & $\begin{array}{l}\text { nosocomial infections } \\
18.2 \% \text { vs } 15.2 \% \text { n.s. }\end{array}$ & $\begin{array}{l}\text { No } \\
\text { effect }\end{array}$ & $\begin{array}{l}\text { ARDS-free survival, } \\
\text { MOF, mortality } \\
29.1 \% \text { vs } 22.2 \% \text { n. } \\
\text { s. }\end{array}$ & $\begin{array}{l}\text { No } \\
\text { effect }\end{array}$ & & \\
\hline & $\begin{array}{l}\text { Immuno- } \\
\text { globulin }\end{array}$ & $\begin{array}{l}\text { Glinz et al, } \\
1985 \text { [20] }\end{array}$ & $\begin{array}{l}\text { any } 47 \% \text { vs } 68 \% P= \\
0.02, \text { pneumonia } 37 \% \text { vs } \\
58 \% P=0.01 \text {, sepsis } 18 \% \\
\text { vs } 26 \% \text { n.s. }\end{array}$ & $\downarrow$ & $\begin{array}{l}\text { Mortality from } \\
\text { infection* } 12 \% \text { vs } \\
11 \% \text { n.s. }\end{array}$ & $\begin{array}{l}\text { No } \\
\text { effect }\end{array}$ & acute phase proteins n.s. & $\begin{array}{l}\text { No } \\
\text { effect }\end{array}$ \\
\hline & & $\begin{array}{l}\text { Douzinas } \\
\text { et al, } 2000 \\
{[32]}\end{array}$ & $\begin{array}{l}\text { pneumonia } 10 \% \text { vs } 61 \% \\
P=0.003\end{array}$ & $\downarrow$ & $\begin{array}{l}\text { Mortality rom } \\
\text { infection* } 0 \text { vs } 0\end{array}$ & $\begin{array}{l}\text { No } \\
\text { effect }\end{array}$ & $\begin{array}{l}\text { C3 and CH50 n.S., C4 increased } \\
p=0.04 \text {, increased serum } \\
\text { bactericidal activity } P< \\
0.000001\end{array}$ & CARS $\downarrow$ \\
\hline & IFN- $\gamma$ & $\begin{array}{l}\text { Polk et al, } \\
1992 \text { [22] }\end{array}$ & $\begin{array}{l}\text { major } 39 \% \text { vs } 35 \% \text {, } \\
\text { minor } 20 \% \text { vs } 28 \% \text {, } \\
\text { pneumonia } 27 \% \text { vs } 24 \% \\
\text { n.s. }\end{array}$ & $\begin{array}{l}\text { No } \\
\text { effect }\end{array}$ & $\begin{array}{l}\text { Mortality } 9.2 \% \text { vs } \\
12.5 \% \text { n.s. }\end{array}$ & $\begin{array}{l}\text { No } \\
\text { effect }\end{array}$ & HLA-DR increased $P=0.0001$ & CARS $\downarrow$ \\
\hline & & $\begin{array}{l}\text { Livingston } \\
\text { et al, } 1994 \\
{[31]}\end{array}$ & $\begin{array}{l}\text { major infection } 48 \% \text { vs } \\
31 \% \text { n.s. }\end{array}$ & $\begin{array}{l}\text { No } \\
\text { effect }\end{array}$ & & & $\begin{array}{l}\text { WBC decreased } P<0.05 \text {, HLA- } \\
\text { DR increased } P<0.05\end{array}$ & $\begin{array}{l}\text { SIRS } \downarrow \\
\text { and } \\
\text { CARS } \downarrow\end{array}$ \\
\hline & & $\begin{array}{l}\text { Dries et al, } \\
1998[18]\end{array}$ & $\begin{array}{l}\text { major infection } 49 \% \text { vs } \\
58 \% \text { n.s. }\end{array}$ & $\begin{array}{l}\text { No } \\
\text { effect }\end{array}$ & $\begin{array}{l}\text { Mortality } 13 \% \text { vs } \\
42 \% P=0.017\end{array}$ & $\downarrow$ & TNF $\alpha, I L-1 \beta, I L-2, I L-4, I L-6$ n.s. & $\begin{array}{l}\text { No } \\
\text { effect }\end{array}$ \\
\hline & & $\begin{array}{l}\text { Nakos et } \\
\text { al, 2002 } \\
{[26]}\end{array}$ & $\begin{array}{l}\text { ventilator-associated } \\
\text { pneumonia } 9 \% \text { vs 50\% } \\
p<0.05\end{array}$ & $\downarrow$ & $\begin{array}{l}\text { Mortality } 27 \% \text { vs } \\
40 \% \text { n.s. }\end{array}$ & $\begin{array}{l}\text { No } \\
\text { effect }\end{array}$ & $\begin{array}{l}\text { HLA-DR expression, IL-1 } \beta \text {, } \\
\text { phospholipase } A 2 \text { all increased } P \\
<0.05 \text {; total cells in BAL and IL- } \\
10 \text { decreased } P<0.01\end{array}$ & $\begin{array}{l}\text { SIRS } \downarrow \\
\text { and } \\
\text { CARS } \downarrow\end{array}$ \\
\hline & Glucan & $\begin{array}{l}\text { Browder } \\
\text { et al, } 1990 \\
{[29]}\end{array}$ & $\begin{array}{l}\text { sepsis } 9.5 \% \text { vs } 49 \% P< \\
0.05\end{array}$ & $\downarrow$ & $\begin{array}{l}\text { Mortality from } \\
\text { sepsis* } 0 \text { vs } 18 \% \\
\text { n.s. }\end{array}$ & $\begin{array}{l}\text { No } \\
\text { effect }\end{array}$ & $\begin{array}{l}\text { IL-1 } \beta \text { decreased } P<0.05, \text { TNF } \alpha \\
\text { n.s. }\end{array}$ & $\overline{S I R S \downarrow}$ \\
\hline & & $\begin{array}{l}\text { de Felippe } \\
\text { et al, } 1993 \\
{[30]}\end{array}$ & $\begin{array}{l}\text { pneumonia } 9.5 \% \text { vs } 55 \% \\
P<0.01 \text {, sepsis } 9.9 \% \text { vs } \\
35 \% P<0.05 \text {, either or } \\
\text { both } 14.3 \% \text { vs } 65 \% P< \\
0.001\end{array}$ & $\downarrow$ & $\begin{array}{l}\text { Mortality: general } \\
23.5 \% \text { vs } 42.1 \% \text {, } \\
\text { related to } \\
\text { infection } 4.8 \% \text { vs } \\
30 \% P<0.05\end{array}$ & $\downarrow$ & & \\
\hline \multirow[t]{5}{*}{$\begin{array}{l}\text { Reduce } \\
\text { hyper } \\
\text { inflammation }\end{array}$} & $\begin{array}{l}\text { Superoxide } \\
\text { dismutase }\end{array}$ & $\begin{array}{l}\text { Marzi et al, } \\
1993[25]\end{array}$ & & & $\begin{array}{l}\text { Mortality } 17 \% \text { vs } \\
8.3 \% \text { n.s. MOF } \\
\text { score n.s. }\end{array}$ & $\begin{array}{l}\text { No } \\
\text { effect }\end{array}$ & $\begin{array}{l}\text { WBC count, CRP, PMN-elastase } \\
\text { and IL- } 6 \text { n.S.; phospholipase A2 } \\
\text { and conjugated dienes } \\
\text { decreased } P<0.05\end{array}$ & $\overline{S I R S \downarrow}$ \\
\hline & $\begin{array}{l}\text { Antithrombin } \\
\text { III }\end{array}$ & $\begin{array}{l}\text { Waydhas } \\
\text { et al, } 1998 \\
{[28]}\end{array}$ & & & $\begin{array}{l}\text { Mortality } 15 \% \text { vs } \\
5 \% \text {, MOF } 20 \% \text { vs } \\
30 \% \text { n.s }\end{array}$ & $\begin{array}{l}\text { No } \\
\text { effect }\end{array}$ & $\begin{array}{l}\text { soluble TNF receptor II, } \\
\text { neutrophil elastase, IL-RA, IL-6, } \\
\text { and IL-8 n.s. }\end{array}$ & $\begin{array}{l}\text { No } \\
\text { effect }\end{array}$ \\
\hline & Anti-CD18 & $\begin{array}{l}\text { Rhee et al, } \\
2000 \text { [23] }\end{array}$ & $\begin{array}{l}\text { major and minor } 38 \% \text { vs } \\
40 \% \text { n.s. }\end{array}$ & $\begin{array}{l}\text { No } \\
\text { effect }\end{array}$ & $\begin{array}{l}\text { Mortality } 5.8 \% \text { vs } \\
6.7 \% \text {, MOF score } \\
\text { n.s. }\end{array}$ & $\begin{array}{l}\text { No } \\
\text { effect }\end{array}$ & $\begin{array}{l}\text { WBC increased } P \text {-value not } \\
\text { reported }\end{array}$ & $\mathrm{SIRS} \uparrow$ \\
\hline & Anti-L-Selectin & $\begin{array}{l}\text { Seekamp } \\
\text { et al, } 2004 \\
{[16]}\end{array}$ & $67 \%$ vs $55 \%$ n.s. & $\begin{array}{l}\text { No } \\
\text { effect }\end{array}$ & $\begin{array}{l}\text { MOF n.s., mortality } \\
11 \% \text { vs } 25 \% \text { n.s. }\end{array}$ & $\begin{array}{l}\text { No } \\
\text { effect }\end{array}$ & $\begin{array}{l}\text { WBC, IL-6, IL-10, neutrophil } \\
\text { elastase, C3a, procalcitonin n.s. }\end{array}$ & $\begin{array}{l}\text { No } \\
\text { effect }\end{array}$ \\
\hline & $\begin{array}{l}\text { Leukoreduced } \\
\text { blood }\end{array}$ & $\begin{array}{l}\text { Nathens et } \\
\text { al, } 2006 \\
{[21]}\end{array}$ & $30 \%$ vs $36 \%$ n.s. & $\begin{array}{l}\text { No } \\
\text { effect }\end{array}$ & $\begin{array}{l}\text { Mortality } 19 \% \text { vs } \\
15 \% \text { n.s. MOF } \\
\text { score } 6.6 \text { vs } \\
5.9 \text { n.s. }\end{array}$ & $\begin{array}{l}\text { No } \\
\text { effect }\end{array}$ & & \\
\hline
\end{tabular}


Table 3 Study results (Continued)

\begin{tabular}{|c|c|c|c|c|c|c|c|}
\hline Perflubron & $\begin{array}{l}\text { Croce } \\
\text { et al, } 1998 \\
{[24]}\end{array}$ & $\begin{array}{l}\text { pneumonia } 50 \% \text { vs } 3 \\
75 \% \text { n.s. }\end{array}$ & $\begin{array}{c}\text { No } \\
\text { effect }\end{array}$ & $\begin{array}{l}\text { Mortality } 8.3 \% \text { vs } \\
25 \% \text { n.s. }\end{array}$ & $\begin{array}{l}\text { No } \\
\text { effect }\end{array}$ & $\begin{array}{l}\text { WBC, neutrophils, IL-6, and IL-10 } \\
\text { all decreased } p<0.01 \text {; capillary } \\
\text { leak (BAL protein), TNF } \alpha, I L-1 \beta \text {, } \\
\text { and IL-8 n.s. }\end{array}$ & SIRS $\downarrow$ \\
\hline $\begin{array}{c}\text { Prostaglandin } \\
\text { E1 }\end{array}$ & $\begin{array}{l}\text { Vassar } \\
\text { et al, } 1991 \\
{[15]}\end{array}$ & $\begin{array}{l}\text { sepsis } 28 \% \text { vs } 30 \% \text {, } \\
\text { major wound inf. } 65 \% \text { vs } \\
72 \% \text {, n.s. }\end{array}$ & $\begin{array}{l}\text { No } \\
\text { effect }\end{array}$ & $\begin{array}{l}\text { Mortality } 26 \% \text { vs } \\
28 \% \text {, ARDS } 13 \% \text { vs } \\
32 \% \text {, MOF } 30 \% \text { vs } \\
32 \% \text { n.s. }\end{array}$ & $\begin{array}{l}\text { No } \\
\text { effect }\end{array}$ & $\begin{array}{l}\text { PMN superoxide production } \\
\text { increased toward normal } P< \\
0.02\end{array}$ & CARS $\downarrow$ \\
\hline
\end{tabular}

ARDS, acute respiratory distress syndrome; CARS, compensatory anti-inflammatory response syndrome; CRP, C-reactive protein; HLA, human leukocyte antigen; IL, interleukin; MOF, multiple organ failure; n.s., not significant; PMN, polymorphnuclear; SIRS, systemic inflammatory response syndrome; TNF, tumor necrosis factor; *, excluding deaths from cardiac arrhythmias secondary to a pulmonary embolus and myocardial infarction, intracranial pressure, and tracheostomy.

the longest follow-up, and did not differ from the other studies regarding the ages or ISS of the patients. Besides the test intervention, only the duration of the test intervention distinguished the studies that reported a significant efficacy in preventing adverse clinical outcome from those that did not; none of the single-dose interventions proved efficacious $[16,17,19,23,27]$.

\section{Discussion}

Although posttraumatic immune deregulation is apparent, the solution is not. In this systematic review we show that administration of immunomodulative interventions often leads to beneficial changes in the inflammatory response. Only administration of immunoglobulin, IFN- $\gamma$, or glucan was efficacious in reducing infection and/or mortality rate.

Immunoglobulin and IFN- $\gamma$ both increase the antigenpresenting capacity of the host. After injury, circulating IgG levels are decreased [32]. Administration of exogenous immunoglobulins results in normalization of IgG concentrations and thus increases IgG-mediated antigen presentation. IgG is a plasma product obtained from healthy donors. IgG was given in the mentioned studies at a dose of 0.25 to $1.0 \mathrm{~g} / \mathrm{kg}$ intravenously and reduced infections in trauma patients, which was more clearly seen in combination with antibiotics $[20,32]$. IFN- $\gamma$ increases antigen presentation to lymphocytes via induction of HLA-DR expression on monocytes. Recombinant IFN- $\gamma$ was given daily at a dose of $100 \mu \mathrm{g}$ subcutaneously $[18,22,26,31]$, but only had an positive effect on mortality [18] and infection [26] in two of four studies. Glucan, a component of the inner cell wall of Saccharomycces cerevisiae, reduces the immune paralysis via a different manner. It decreases prostaglandin release by macrophages but also stimulates bone marrow proliferation [29]. This bone marrow proliferation may be in favor in the late immune paralysis. Glucan was given at a dose of $50 \mathrm{mg} / \mathrm{m}^{2}$ daily [29] or $30 \mathrm{mg}$ every 12 hours [30], resulting in a reduced infection and mortality rate. All these seemingly effective interventions started on the day of admission and were continued until at least three to seven days after trauma.
As every systematic review, this study has its restrictions. A clear limitation of the trials is their relatively small sample size and the heterogeneity of interventions and study populations. Furthermore, we can not completely rule out publication bias. Yet, none of the studies report financial support by a pharmaceutical company and some studies show a negative result. Also, no other studies with immunoglobulin, IFN $-\gamma$, or glucan in trauma patients were found searching the clinical trial register database [33].

Challenges unique to the trauma population impede designing large RCTs. Polk et al. [22] note that patient homogeneity is difficult to achieve in multicenter trials because different centers tend to receive different patients. In addition, in the rush of the emergency care of severely injured patients, informed consent must wait until a family member is contacted [23] whereas the initiation of treatment cannot wait. Bulger et al. [19], Nathens et al. [21], and Rizoli et al. [27] solved this problem by gaining permission from their ethics committees to delay informed consent until after the initial treatment, but this approach is not always accepted. Furthermore, assessing patient eligibility for inclusion in the trial is time consuming. Delay to randomize patients can be avoided by using simple inclusion criteria. Nathens et al. [21] used only one criterion, the request of the physician for red blood cells for an expected transfusion, but were then faced with the possible dilution of treatment effect when they performed an intention-to-treat analysis because many randomized patients never received any blood products.

Based on the selected studies, general conclusions regarding the efficacy of potentially immunomodulative interventions cannot be drawn. As explained in the results section, the intended effects of the interventions on the inflammatory response differed. Furthermore, data from pilot studies [14,24-27] and phase II trials $[16,23,24]$ should be used to steer future investigations rather than to draw definitive conclusions. Interventions that did not have a significant effect on clinical outcome may need to be administered earlier [25], continued longer $[16,22,25,28]$, or need sequential specific timing 
to be effective [22]. Seekamp et al. [16] and Rhee et al. [23] explicitly chose for a single dose of an anti-inflammatory cytokine because they wanted to taper the initial hyper-inflammatory response without compounding the later immune paralysis. Timing is essential in accurate modulation of the immune response after trauma. The lack of a positive effect can be the result of wrong timing rather that to the drug itself. Consequently differences in timing between interventional drugs studied in this systematic review may contribute to disparity in outcome.

Besides changing timing, some authors recommended the use of larger doses $[19,28]$. Waydhas et al. [28] suggest that concomitant heparinization interfered with the immunomodulative effect of antithrombin III. The use of these drugs is inevitable in severely injured patients. Where theoretically promising approaches did not produce the results hoped for, sufficiently powered phase IV trails are needed.

Another impediment for drawing general conclusions is the fact that study populations differed greatly across the studies. For example, although Croce et al. [24] excluded patients with injuries thought to be lethal within 30 days of injury, others only excluded patients when the injuries were thought to be lethal within only one [28], two $[16,20,21,23]$, or five [30] days. Similarly, de Felippe et al. [30] only included patients with concomitant head injury, whereas other researchers excluded patients with major head injury $[16,19,23,28]$ or any head injury $[14,29]$. Mortality by severe head injury or massive bleeding may mask the effect of the interventional drug in an intention-to-treat trial, especially in trials with a small sample size.

Some researchers chose to exclude patients receiving steroids $[24,25,31,32]$, because the efficacy of immunomodulative interventions is likely to be affected by simultaneous administration of steroids and/or antibiotics during care-as-usual [32]. However, this approach leads to a selection bias including patients that are more likely to have a favorable outcome.

Patient selection is imperative. Where no significant benefit was found for the test group as a whole, study authors postulated more specific inclusion criteria were necessary for future studies. For example, older patients $[19,24,26]$, those with more severe injuries $[19,23,26]$, patients needing 10 or more units of packed red blood cells [24], and those who had a longer time from injury to enrollment in the study [24] were more susceptible to organ dysfunction and thus likely to benefit more from immunomodulative intervention. Selection of patients at risk may favor the outcome where no significant difference was found in a broader group of patients. Researchers suggest future study participants be select based not only the injury severity, but also on sepsis [28] or inflammatory parameters [16] as Nakos et al. [26] did when they only randomized patients after ascertaining immune paralysis by measuring the HLADR in bronchoalveolar lavage.

Interpretations of the efficacy of immune modulating therapies in trauma patients remains difficult. More studies with similar study populations will aid comparison of the effect of different interventions in trauma patients.

\section{Conclusions}

An array of potentially immunomodulative interventions have been tested in a heterogeneous group of trauma patients in level $1 \mathrm{~b}$ and $2 \mathrm{~b}$ RCTs. Reported changes in inflammatory parameters could indicate an attenuation of SIRS and/or CARS; however, they were not consistently accompanied by significant changes in infection and mortality rates. Administation of immunoglobulin, IFN- $\gamma$, and glucan was efficacious whereas none of the single-dose interventions were. Further trials powered to measure efficacy may reveal which immunomodulative interventions should be routinely implemented to save lives of trauma patients.

\section{Key messages}

- Inflammatory complications, such as MOF and severe infection, are the most common cause of late death in trauma patients.

- An array of potentially immunomodulative interventions have been tested in a heterogeneous group of trauma patients in RCTs.

- Extensive disparity in study populations impairs inter-trial evaluation of efficacy of different (immunomodulative) interventions. Therefore, more standardized inclusion criteria are recommended.

- In most studies, the inflammatory parameters differed significantly between the test and control groups. However, significant changes in infection, MOF, and mortality rates were only measured in studies testing immunoglobulin, IFN- $\gamma$, and glucan.

- A recommendation can be made to administer immunoglobulin, IFN- $\gamma$ or glucan to improve the outcome of trauma patients.

\section{Abbreviations}

CARS: compensatory anti-inflammatory response syndrome; HLA: human leukocyte antigen; IFN: interferon; IL: interleukin; ISS: injury severity score; MARS: mixed antagonistic response syndrome; MOF: multiple organ failure; RCT: randomized controlled trial; SIRS: systemic inflammatory response syndrome; TNF: tumor necrosis factor.

\section{Authors' contributions}

NS and LL conceived of and designed the study. NS and TV were involved in data acquisition, analysis, and interpretation and drafted the manuscript. $\mathrm{LL}$ and TV critically revised the manuscript for important intellectual content. All authors read and approved the final manuscript. 
Authors' information

NS and TV are PhD students at the Department of Surgery. LL is the Department's Professor of Traumatology.

\section{Competing interests}

The authors declare that they have no competing interests.

Received: 26 February 2010 Revised: 6 May 2010

Accepted: 5 August 2010 Published: 5 August 2010

\section{References}

1. Peden M, McGee K, Krug E: Injury: A leading cause of the global burden of disease, 2000. Geneva, Switzerland: World Health Organization 2002.

2. Teixeira PG, Inaba K, Hadjizacharia P, Brown C, Salim A, Rhee P, Browder T, Noguchi TT, Demetriades D: Preventable or potentially preventable mortality at a mature trauma center. J Trauma 2007, 63:1338-1346, discussion 1346-1337.

3. Moore FA, Sauaia A, Moore EE, Haenel JB, Burch JM, Lezotte DC: Postinjury multiple organ failure: a bimodal phenomenon. J Trauma 1996, 40:501-510, discussion 510-502.

4. Bone RC: Sir Isaac Newton, sepsis, SIRS, and CARS. Crit Care Med 1996, 24:1125-1128

5. Hietbrink F, Koenderman L, Rijkers $G$, Leenen L: Trauma: the role of the innate immune system. World J Emerg Surg 2006, 1:15.

6. Keel M, Trentz O: Pathophysiology of polytrauma. Injury 2005, 36:691-709.

7. Moore EE, Moore FA, Harken AH, Johnson JL, Ciesla D, Banerjee A: The two-event construct of postinjury multiple organ failure. Shock 2005, 24:71-74.

8. Montejo JC, Zarazaga A, Lopez-Martinez J, Urrutia G, Roque M, Blesa AL, Celaya S, Conejero R, Galban C, Garcia de Lorenzo A, Grau T, Mesejo A, Ortiz-Leyba C, Planas M, Ordonez J, Jimenez FJ: Immunonutrition in the intensive care unit. A systematic review and consensus statement. Clin Nutr 2003, 22:221-233.

9. Oxford Centre for Evidence-based Medicine - Critical Appraisal. [http:// www.cebm.net/index.aspx?o=1157].

10. Oxford Centre for Evidence-based Medicine - Levels of Evidence (March 2009). [http://www.cebm.net/index.aspx?o=1025].

11. Bauer M, Redl H, Mari I: Prophylactic veno-venous haemofiltration and inflammatory response to multiple trauma. International Journal of Intensive Care 2001, 8:194-199.

12. Rommelsheim K: Preventive use of Pentaglobin in intensive care treatment of trauma patients. Anasth Intensivther Notfallmed 1989, 24:162-166.

13. Dries DJ, Jurkovich GJ, Maier RV, Clemmer TP, Struve SN, Weigelt JA, Stanford GG, Herr DL, Champion HR, Lewis FR, et al: Effect of interferon gamma on infection-related death in patients with severe injuries. A randomized, double-blind, placebo-controlled trial. Arch Surg 1994, 129:1031-1041, discussion 1042

14. Miller $C L$, Lim RC: Dextran as a modulator of immune and coagulation activities in trauma patients. J Surg Res 1985, 39:183-191.

15. Vassar MJ, Fletcher MP, Perry CA, Holcroft JW: Evaluation of prostaglandin E1 for prevention of respiratory failure in high risk trauma patients: a prospective clinical trial and correlation with plasma suppressive factors for neutrophil activation. Prostaglandins Leukot Essent Fatty Acids 1991, 44:223-231.

16. Seekamp A, Van Griensven M, Dhondt E, Diefenbeck M, Demeyer I, Vundelinckx G, Haas N, Schaechinger U, Wolowicka L, Rammelt S, Stroobants J, Marzi I, Brambrink AM, Dziurdzik P, Gasiorowski J, Redl H, Beckert M, Khan-Boluki J: The effect of anti-L-selectin (aselizumab) in multiple traumatized patients - Results of a phase II clinical trial. Crit Care Med 2004, 32:2021-2028.

17. Seekamp A, Van Griensven M, Rusu C, Konig J, Khan-Boluki J, Redl H: The effect of anti-L-selectin (Aselizumab) on the posttraumatic inflammatory response in multiply traumatized patients. European Journal of Trauma 2005, 31:557-567.

18. Dries DJ, Walenga JM, Hoppensteadt D, Fareed J: Molecular markers of hemostatic activation and inflammation following major injury: effect of therapy with IFN-gamma. J Interferon Cytokine Res 1998, 18:327-335.

19. Bulger EM, Jurkovich GJ, Nathens AB, Copass MK, Hanson S, Cooper C, Liu PY, Neff M, Awan AB, Warner K, Maier RV: Hypertonic resuscitation of hypovolemic shock after blunt trauma: a randomized controlled trial. Arch Surg 2008, 143:139-148, discussion 149.

20. Glinz W, Grob PJ, Nydegger UE, Ricklin T, Stamm F, Stoffel D, Lasance A: Polyvalent immunoglobulins for prophylaxis of bacterial infections in patients following multiple trauma. A randomized, placebo-controlled study. Intensive Care Med 1985, 11:288-294.

21. Nathens AB, Nester TA, Rubenfeld GD, Nirula R, Gernsheimer TB: The effects of leukoreduced blood transfusion on infection risk following injury: a randomized controlled trial. Shock 2006, 26:342-347.

22. Polk HC Jr, Cheadle WG, Livingston DH, Rodriguez JL, Starko KM, Izu AE, Jaffe HS, Sonnenfeld G: A randomized prospective clinical trial to determine the efficacy of interferon-gamma in severely injured patients. Am J Surg 1992, 163:191-196.

23. Rhee P, Morris J, Durham R, Hauser C, Cipolle M, Wilson R, Luchette F, McSwain N, Miller R: Recombinant humanized monoclonal antibody against CD18 (rhuMAb CD18) in traumatic hemorrhagic shock: results of a phase II clinical trial. Traumatic Shock Group. J Trauma 2000, 49:611-619, discussion 619-620.

24. Croce MA, Fabian TC, Patton JH Jr, Melton SM, Moore M, Trenthem LL: Partial liquid ventilation decreases the inflammatory response in the alveolar environment of trauma patients. J Trauma 1998, 45:273-280, discussion 280-272.

25. Marzi I, Buhren V, Schuttler A, Trentz O: Value of superoxide dismutase for prevention of multiple organ failure after multiple trauma. J Trauma 1993, 35:110-119, discussion 119-120.

26. Nakos G, Malamou-Mitsi VD, Lachana A, Karassavoglou A, Kitsiouli E, Agnandi N, Lekka ME: Immunoparalysis in patients with severe trauma and the effect of inhaled interferon-gamma. Crit Care Med 2002, 30:1488-1494.

27. Rizoli SB, Rhind SG, Shek PN, Inaba K, Filips D, Tien H, Brenneman F, Rotstein $\mathrm{O}$ : The immunomodulatory effects of hypertonic saline resuscitation in patients sustaining traumatic hemorrhagic shock: a randomized, controlled, double-blinded trial. Ann Surg 2006, 243:47-57.

28. Waydhas C, Nast-Kolb D, Gippner-Steppert C, Trupka A, Pfundstein C, Schweiberer L, Jochum M: High-dose antithrombin III treatment of severely injured patients: results of a prospective study. J Trauma 1998, 45:931-940.

29. Browder W, Williams D, Pretus H, Olivero G, Enrichens F, Mao P, Franchello A: Beneficial effect of enhanced macrophage function in the trauma patient. Ann Surg 1990, 211:605-612, discussion 612-603.

30. de Felippe Júnior J, da Rocha e Silva Júnior M, Maciel FM, Soares Ade M, Mendes NF: Infection prevention in patients with severe multiple trauma with the immunomodulator beta 1-3 polyglucose (glucan). Surg Gynecol Obstet 1993, 177:383-388.

31. Livingston DH, Loder PA, Kramer SM, Gibson UE, Polk HC Jr: Interferon gamma administration increases monocyte HLA-DR antigen expression but not endogenous interferon production. Arch Surg 1994, 129:172-178.

32. Douzinas EE, Pitaridis MT, Louris G, Andrianakis I, Katsouyanni K, Karmpaliotis D, Economidou J, Sfyras D, Roussos C: Prevention of infection in multiple trauma patients by high-dose intravenous immunoglobulins. Crit Care Med 2000, 28:8-15.

33. Clinicaltrial.gov. [http://www.clinicaltrial.gov].

doi:10.1186/cc9218

Cite this article as: Spruijt et al:: A systematic review of randomized controlled trials exploring the effect of immunomodulative interventions on infection, organ failure, and mortality in trauma patients. Critical Care 2010 14:R150. 